\title{
Aplicação da luva funcional em um indivíduo com paralisia de mão e punho: um estudo piloto*
}

\section{Application of functional glove in an individual with paralysis of the hand and wrist: a pilot study}

\author{
Kátia Vanessa Pinto de Meneses ${ }^{1}$, Daniel Neves Rocha ${ }^{2}$, Maurício \\ Ferrari Santos Corrêa ${ }^{3}$, Marcos Pinotti ${ }^{4}$
}

\begin{abstract}
MENESES, K. V. P. de; ROCHA, D. N.; CORRÊA, M. F. S.; PINOTTI, M. Aplicação da luva funcional em um indivíduo com paralisia de mão e punho: um estudo piloto. Rev. Ter. Ocup. Univ. São Paulo, v. 20, n. 2, p. 92-100, maio/ago. 2009.

RESUMO: Uma das intervenções bastante utilizadas pelo terapeuta ocupacional na reabilitação de indivíduos com disfunções físicas é a fabricação e a prescrição de órteses. Porém, órteses funcionais para mão tem se mostrado com um dos grandes desafios do processo de reabilitação devido à grande complexidade da mão humana. A motivação para este trabalho se deu a partir dos resultados dos testes in vitro realizados com o protótipo da Luva Funcional, que apresentou grande potencial em possibilitar a flexão e extensão dos dedos em indivíduos com paralisia na mão. O objetivo deste estudo foi verificar o uso da Luva Funcional em um indivíduo que não apresentasse movimentos ativos em mão e punho. Foi realizado um estudo piloto, com aplicação dos seguintes intrumentos: Teste para Desempenho em Atividades Unimanuais, Teste para Desempenho em Atividades Bimanuais e Questionário de Satisfação. A Luva Funcional foi capaz de possibilitar os movimentos de flexão e de extensão dos dedos em um indivíduo com paralisia em mão e punho devido à lesão do plexo braquial. O paciente conseguiu controlar a abertura e fechamento da mão por meio do sinal mioelétrico dos músculos selecionados para sua ativação e conseguiu desempenhar, com o uso da órtese Luva funcional, atividades unimanuais e bimanuais. O resultado do Questionário de Satisfação demonstrou satisfação do paciente com o uso da órtese Luva Funcional.
\end{abstract}

DESCRITORES: Equipamentos de atuo-ajuda/utilização. Neuropatias do plexo brachial/ reabilitação. Tecnologia/métodos. Terapia ocupacional.

\footnotetext{
* Este trabalho é parte da tese de doutorado: Avaliação funcional de uma órtese para mão com músculos artificiais eletromecânicos em pacientes com lesão de plexo braquial (MENESES, 2008). Parte dos resultados aqui trazidos foi divulgada nos seguintes eventos: Technology and Medical Sciences International em Diamantina (2008), 28 Congresso Brasileiro de Cirurgia da Mão em Ribeirão Preto (2008) e no 2 Encontro de Tecnologia Assistiva da FMRP-USP em Ribeirão Preto (2008).

1. Terapeuta Ocupacional, mestre e doutora em Bioengenharia pela UFMG.

2. Engenheiro, mestre em Engenharia Mecânica pela UFMG, doutorando em Engenharia Mecânica pela UFMG.

${ }^{3}$. Aluno de graduação em Engenharia Mecânica da UFMG.

${ }^{4}$. Docente do Departamento de Engenharia Mecânica da UFMG; Coordenador do Laboratório de Bioengenharia da UFMG.

Endereço para correspondência: Rua Avenida Flamboyant, Residencial Flamboyant, lote 22, bloco C, apto 1001, Aguas Claras, DF. CEP 71917-000.katiavpmeneses@gmail.com
} 


\section{INTRODUÇÃO}

prescrição, projeto, fabricação, verificação e
treino de órteses são parte das intervenções
utilizadas pelo terapeuta ocupacional com indivíduos apresentando disfunções físicas, com objetivo de recuperar a função e melhorar o desempenho ocupacional.

Segundo Trombly e Liden (1995), órtese é um dispositivo que se acrescenta ao corpo para substituir um poder motor ausente, para restaurar a função, auxiliar músculos fracos, posicionar ou mobilizar uma articulação ou corrigir deformidades. As órteses são tradicionalmente classificadas em estáticas, dinâmicas e funcionais. Órteses estáticas imobilizam ou repousam uma articulação ou membro, não apresentando partes móveis. Essas órteses são usadas para proteger uma articulação, como no póscirúrgico, para prevenção de deformidades, como em queimaduras e artrite reumatóide, podendo também ser usadas para correção e alinhamento articular. Órteses dinâmicas proporcionam movimentos por meio de molas, elásticos ou outros materiais. Esses dispositivos podem substituir uma musculatura ausente, auxiliar músculos fracos, prevenir ou corrigir contraturas, manter o equilíbrio de forças (paralisias), promover repouso (cicatrização, infecção, dor) ou mobilizar articulações específicas (manter ou aumentar amplitude de movimento). Órteses funcionais podem ser dinâmicas ou estáticas e tem por objetivo possibilitar a função.

Órteses funcionais para mão tem se mostrado com um dos grandes desafios do processo de reabilitação devido à grande complexidade da mão humana. As poucas órteses funcionais existentes são, em sua maioria para o braço (KOHLMEYER et al., 1990; PERRY, 1974) sendo raras as órteses funcionais para mão que, por sua vez, foram desenvolvidas para atender pacientes tetraplégicos (ALON, 2003; BENJUYA; KENNEY, 1990; PINTO, 1999; PROCHAZKA et al., 1997, WIERZBICKA; WIEGNER, 1996). Estas usam do movimento do punho para potencializar o movimento dos dedos (tenodese) ou exigem a contração ativa de músculos localizados no antebraço (TROMBLY; RANDOMSKI, 2005; PINTO, 1999; HARVEY, 1996; KILGORE et al., 1989), não podendo ser usadas por indivíduos com ausência destes movimentos ativos, como acontece na maioria dos tipos de lesões do plexo braquial. Outro problema, é que geralmente as órteses são confeccionadas de materiais rígidos, apresentam grande volume, e são pouco aceitáveis esteticamente. Além disto, a maior parte dessas órteses são complexas em termos de mecânica e controle (PETROFF et al., 2001; ROMILLY et al., 1994; SLACK; BERBRAYER, 1992); são importadas e, não raro, de alto custo, fazendo com que esteja muito além da disponibilidade financeira da maioria dos pacientes no Brasil.

Diante da necessidade de órteses mais eficázes, capazes de possibilitar a função na mão, foi desenvolvido um novo protótipo de órtese (ROCHA, 2007; ROCHA et al., 2007; MENESES, 2005; MENESES et al., 2005) conhecida como Luva Funcional. A Luva Funcional é composta por uma luva de tecido acoplada a tendões artificiais, uma tala para posicionamento da articulação do punho, um motor, um circuito de controle e eletrodos de superfíce. $\mathrm{O}$ acionamento é realizado por meio da captação de sinais mioelétricos de músculos previamente selecionados.

A motivação para este trabalho se deu a partir dos resultados dos testes in vitro realizados com o protótipo da Luva Funcional (MENESES et. al., 2005, 2008; MENESES, 2005), que apresentou grande potencial em possibilitar a flexão e extensão dos dedos em indivíduos com paralisia na mão.

O objetivo deste estudo foi verificar o uso da Luva Funcional em um indivíduo que não apresentasse movimentos ativos em mão e punho.

\section{MATERIAIS E MÉTODOS}

Esta pesquisa foi aprovada pelo Comitê de Ética em Pesquisa da Universidade Federal de Minas Gerais UFMG (Parecer no ETIC 368/06) e pelo Comitê de Ética em Pesquisa da Secretaria Municipal de Saúde de Belo Horizonte (Protocolo 016/2007).

Foi realizado um estudo piloto, onde se selecionou um único paciente com paralisia em punho e mão, devido à lesão do plexo braquial. Para participar da pesquisa era necessário que o indivíduo não apresentasse movimentos ativos em mão e punho devido lesão de plexo braquial unilateral; tempo mínimo de dois anos após a lesão ou após os procedimentos cirúrgicos realizados; quadro neurológico estável (relatório fornecido pelo médico do paciente relatando a ausência de perspectiva de recuperação dos nervos afetados); idade igual ou superior a 18 anos; movimentação passiva completa das articulações do punho, da mão e dos dedos; presença de contração muscular ativa de músculos localizados na cintura escapular ou no membro superior (podendo ser do mesmo lado da lesão ou contralateral) para a ativação da órtese; capacidade de entender aos comandos para o aprendizado do mecanismo de acionamento da órtese. Foram excluídos desta pesquisa indivíduos que apresentaram contraturas articulares e/ou musculares que limitem os movimentos dos dedos; doenças degenerativas progressivas; idade 
inferior a 18 anos; déficit cognitivo que impeça ao paciente a compreensão das orientações quanto ao uso da órtese e seu treinamento; lesões de pele nos locais de colocação da órtese; outras enfermidades que possam interferir com a sua participação na pesquisa.

As avaliações e testes clínicos com a órtese Luva Funcional foram realizados no setor de Terapia Ocupacional do Centro Geral de Reabilitação (CGR), em Belo Horizonte, Minas Gerais. Após a seleção, o participante foi esclarecido sobre os objetivos do estudo e, estando de acordo, assinou o termo de consentimento livre e esclarecido. A Luva Funcional foi confeccionada baseando-se nos moldes da mão do paciente piloto (Figura 1).

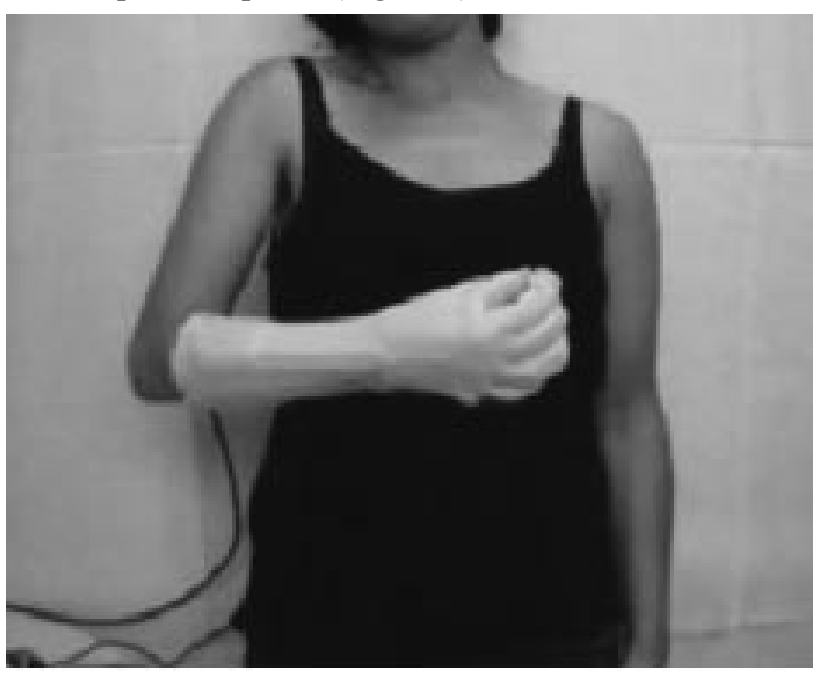

FIGURA 1. Paciente usando a Luva Funcional

$\mathrm{O}$ estudo foi dividido em três fases. A primeira fase teve o objetivo de selecionar a musculatura adequada para acionamento do sistema eletromecânico e realizar o treinamento do sujeito para contrair e relaxar cada grupo muscular de forma individualizada. A segunda fase teve o objetivo de treinar o paciente no uso da órtese Luva Funcional. Na terceira fase foram aplicados instrumentos para testar a função de preensão da mão em atividades unimanuais e bimanuais.

Foi realizada ampla pesquisa na literatura a procura de instrumentos padronizados apropriados para avaliar a função de preensão da mão. A maioria dos testes encontrados avalia a agilidade e destreza da mão, bem como o desempenho em atividades de vida diária, exigindo que o indivíduo realize movimentos ativos em várias articulações do membro superior como ombro, cotovelo, antebraço e punho. Como muitos pacientes com lesão do plexo braquial apresentam ausência de movimentação ativa em várias articulações do membro superior lesado, e visto a necessidade de testar apenas a flexão e extensão dos dedos com o uso da órtese Luva Funcional, estes instrumentos se mostraram inadequados. Frente a esta dificuldade de encontrar instrumentos que avaliam a capacidade de preensão da mão, independente do paciente apresentar ou não função no braço, foram construídos três instrumentos: Teste para Desempenho em Atividades Unimanuais, Teste para Desempenho em Atividades Bimanuais e um Questionário de Satisfação.

Durante os Testes de Desempenho em Atividades Unimanuais e Bimanuais foi utilizada uma tipóia para posicionar o membro superior do paciente em aproximadamente 90 graus de flexão de cotovelo e posição neutra das articulações do ombro (entre rotação interna e externa) e do antebraço (entre pronação e supinação), ficando as articulações do punho e da mão livres. O uso da tipóia teve como objetivo assegurar que fosse avaliada apenas a função da mão, independente do paciente apresentar ou não função no braço.

O Teste para Desempenho em Atividades Unimanuais foi adaptado a partir do "Grasp Release Test" (WUOLLE et al., 1994) que requer que o indivíduo segure, mova e solte cada um de cinco objetos de diferentes formas, pesos e tamanhos. Antes do início do Teste de Desempenho em Atividades Unimanuais, um ensaio preliminar foi administrado de forma a verificar se o paciente entendeu as instruções do teste e verificar a capacidade de segurar cada objeto. Caso o paciente apresentasse dificuldade em manipular algum objeto, era encorajado a praticar até que a manipulação ocorresse com sucesso. Caso fosse verificada a incapacidade de preensão de algum objeto, este seria excluído do teste principal. O teste principal consiste em segurar todos os objetos que obtiveram sucesso no teste preliminar e soltá-los dentro de uma caixa. A Tabela 1. mostra os objetos utilizados neste teste.

Durante o Teste para Desempenho em Atividades Bimanuais o membro superior com a órtese Luva Funcional foi usado como auxiliar para segurar objetos de forma a permitir sua manipulação pela mão dominante (mão preservada). O teste compreendeu cinco tarefas listadas a seguir: 1- Segurar uma folha de papel com a órtese e cortála com uma tesoura usada pela mão dominante; 2- Segurar uma caneta com a órtese e retirar a tampa com a mão dominante; 3-Segurar um copo de plástico rígido vazio com a órtese e enchê-lo com líquido, usando a mão dominante; 4- Segurar um copo de plástico rígido com líquido com a órtese e misturar açúcar segurando uma colher com a mão dominante; 5 - Segurar um recipiente com tampa de rosca (creme dental) com a órtese, retirar a tampa com a mão dominante e colocar creme dental na escova pressionando o tubo. 
MENESES, K. V. P. de et al. Aplicação da luva funcional. Rev. Ter. Ocup. Univ. São Paulo, v. 20, n. 2, p. 92-100, maio/ago. 2009.

TABELA 1. Objetos de teste

\begin{tabular}{llcc}
\hline & \multicolumn{1}{c}{ Objeto } & Massa $(\mathbf{g})$ & Dimensões $(\mathbf{m m})$ \\
\hline 1 & Pino de madeira & 5 & 6 (diâmetro) $\times 76$ \\
2 & Cubo de madeira & 10 & $25 \times 25 \times 25$ \\
3 & Cilindro plástico & 100 & 41 (diâmetro) x 114 \\
4 & Fita de videocassete & 225 & $204 \times 145 \times 25$ \\
5 & Cilindro plástico & 500 & 41 (diâmetro) x 114 \\
\hline
\end{tabular}

Para avaliar a satisfação do indivíduo com o uso da Luva Funcional, foi construído um questionário binomial com questões relativas ao conforto, funcionalidade e satisfação.

Paciente: R.A.S., sexo feminino, 37 anos, sofreu lesão completa de plexo braquial do lado direito (lado dominante), devido a um acidente de bicicleta ocorrido em abril de 2005, apresentando paralisia completa de todo o membro superior direito. Foi submetido à cirurgia para reparo dos nervos em agosto de 2005. Após a cirurgia, obteve retorno de alguns movimentos das articulações do ombro, do cotovelo e do antebraço. Durante avaliação realizada em setembro de 2007 apresentou ativação completa de todos os movimentos escapulares e alguns movimentos ativos da articulação do ombro (10 graus de flexão, 20 graus de abdução e rotação interna completa). Não apresentou movimento ativo de rotação externa de ombro. Na articulação do cotovelo apresentou flexão ativa, com força muscular grau três de acordo com o teste de força manual. Na articulação do antebraço apresentou pronação ativa e ausência completa da supinação. Não apresentou movimentos ativos nas articulações do punho e dedos, porém apresenta movimentação passiva completa destas articulações, conforme exigido nos critérios de inclusão.

\section{RESULTADOS E DISCUSSÃO}

\section{Fase 1: seleção e treinamento da musculatura}

A seleção dos músculos para ativação do sistema eletromecânico foi baseada em estudos anatômicos, cinesiológicos e eletromiográficos com eletrodos de superfície. Buscou-se uma musculatura onde pudessem ser captados sinais mioelétricos distintos entre o estado de relaxamento e o de contração muscular, que fossem de fácil ativação voluntária, além de não interferir nos movimentos de posicionamento do membro superior.

Os músculos selecionados foram trapézio fibras superiores e elevadores de escápula (responsáveis pelo movimento de elevação escapular) e os músculos trapézio fibras médias e rombóides (responsáveis pelo movimento de retração escapular), do lado direito da paciente (Figura 2), mesmo lado do membro lesado.

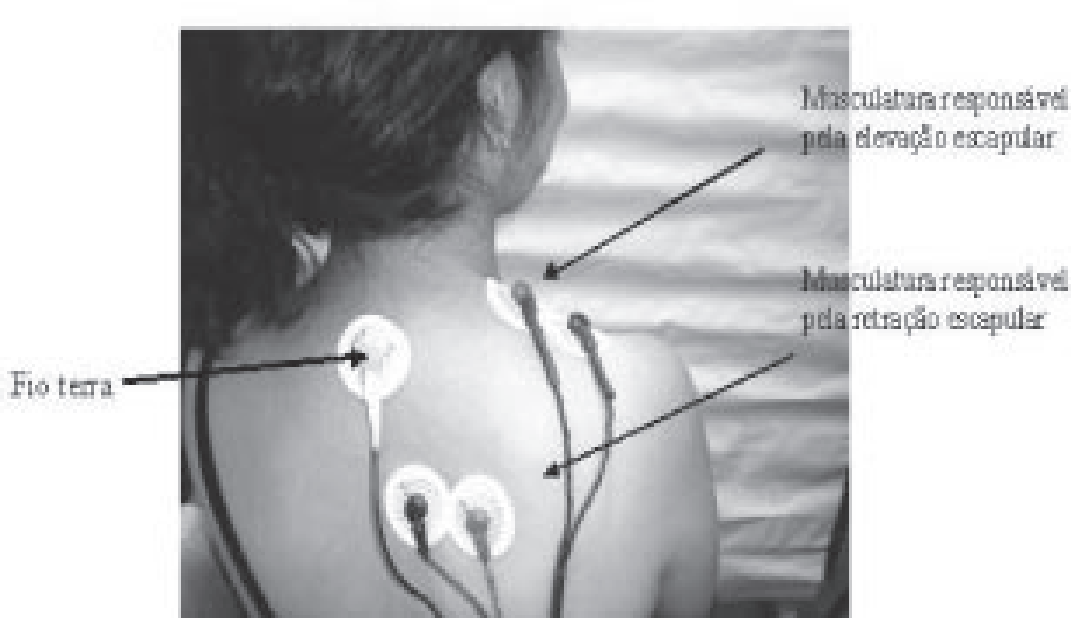

FIGURA 2. Posicionamento dos eletrodos no paciente 
MENESES, K. V. P. de et al. Aplicação da luva funcional. Rev. Ter. Ocup. Univ. São Paulo, v. 20, n. 2, p. 92-100, maio/ago. 2009.

Um Módulo de Treinamento foi desenvolvido para auxiliar o paciente a aprender a controlar a órtese. O Módulo de Treinamento oferece feedback visual, por meio de dois diodos emissores de luz (Light Emitting Diode - LED) de cores diferentes (verde e vermelho), para as ações de abrir e fechar a mão. Quando o paciente ativava a musculatura responsável pela abertura da mão, o LED verde acendia e quando ele contraia a musculatura responsável pelo fechamento da mão o LED vermelho acendia. Se os dois LEDs acendessem, significava que o paciente estava ativando simultaneamente ambos os músculos e, por isso, o sistema não era acionado. Foram utilizados eletrodos de superfície passivos auto-adesivos descartáveis, modelo 2223 (3M, EUA). Nesta fase o paciente aprendeu, em poucos minutos, a contrair e relaxar cada grupo muscular de forma independente, controlando, de forma voluntária, o comando para acender e apagar as luzes do Módulo de Treinamento.

Fase 2: treinar o paciente para com o uso da órtese Luva Funcional

Inicialmente foi solicitado que o paciente realizasse a contração muscular de cada grupo selecionado, para regular a sensibilidade adequada para o sistema de acionamento. O controle da sensibilidade adequada é importante para que se determine o nível de contração muscular desejada para o acionamento do sistema, de forma que contrações involuntárias e resultantes da manutenção postural não interfiram no acionamento do sistema eletromecânico. Depois de regulada a sensibilidade de contração muscular, a luva foi vestida na mão do paciente, conectada ao sistema de acionamento mecânico e sistema elétrico. Foi verificado o ajuste anatômico da Luva Funcional, bem como os pontos de possível pressão. O paciente realizou o correto acionamento da abertura e fechamento da órtese Luva Funcional por meio dos sinais mioelétricos dos músculos previamente selecionados. O paciente apresentou facilidade no controle para abertura e fechamento da Luva Funcional.

\section{Fase 3: aplicação dos instrumentos de teste}

Esta fase teve como objetivo testar o uso da Luva Funcional em atividades unimanuais e bimanuais por meio dos instrumentos de avaliação. O primeiro instrumento a ser utilizado foi o "Teste para Desempenho em Atividades Unimanuais". Um teste preliminar foi administrado para cada objeto a fim de determinar se o paciente entendeu todas as instruções do teste e para selecionar os objetos que fariam parte do teste principal. Durante o teste preliminar o paciente conseguiu manipular todos os objetos sem dificuldades. Portanto, todos os objetos da tabela foram incluídos no teste principal. As instruções para o teste principal foram as seguintes: Segure o pino de madeira e solte-o dentro da caixa; Segure o cubo de madeira e solte-o dentro da caixa; Segure o cilindro de plástico $(100 \mathrm{~g})$ e solte-o dentro da caixa; Segure a fita de videocassete e solte-a dentro da caixa; Segure o cilindro de plástico $(500 \mathrm{~g})$ e solte-o dentro da caixa. O uso da mão contralateral foi permitido apenas para estabilização inicial do objeto. A seqüência de manipulação foi repetida três vezes. Durante o teste preliminar o paciente conseguiu manipular todos os objetos, de forma que todos os objetos foram incluídos nos testes seguintes. Durante os testes 1, 2 e 3, o paciente conseguiu manipular todos os objetos. Os resultados estão listados na Tabela 2 e mostram $100 \%$ de sucesso na manipulação de todos os objetos.

TABELA 2. Resultado do Teste de Desempenho em Atividades Unimanuais

\begin{tabular}{ccccc}
\hline Objeto/Teste & Teste preliminar & Teste 1 & Teste2 & Teste 3 \\
\hline 1 & SIM & SIM & SIM & SIM \\
2 & SIM & SIM & SIM & SIM \\
3 & SIM & SIM & SIM & SIM \\
4 & SIM & SIM & SIM & SIM \\
5 & SIM & SIM & SIM & SIM \\
\hline
\end{tabular}

O segundo instrumento utilizado foi o "Teste para Desempenho em Atividades Bimanuais". A paciente obteve sucesso nas tarefas de 1 a 4 (Tabela 3). Na tarefa de número 5 , a paciente conseguiu segurar o creme dental com a órtese e retirar a tampa com a mão dominante, porém não conseguiu apertar de forma que o creme dental fosse colocado na escova de dente. A Figura 3 mostra a paciente realizando as tarefas de 1 a 5 do Teste de Desempenho em Atividades Bimanuais. 
MENESES, K. V. P. de et al. Aplicação da luva funcional. Rev. Ter. Ocup. Univ. São Paulo, v. 20, n. 2, p. 92-100, maio/ago. 2009.

TABELA 3. Resultado do Teste de Desempenho em Atividades Bimanuais

\begin{tabular}{|c|c|c|c|}
\hline & Tarefa & Sucesso & Insucesso \\
\hline 1 & Segurar folha de papel com a órtese e cortá-la com uma tesoura usada pela mão dominante. & $\mathbf{x}$ & \\
\hline 2 & Segurar caneta com a órtese e retirar a tampa com a mão dominante. & $\mathbf{x}$ & \\
\hline 3 & $\begin{array}{l}\text { Segurar copo de plástico rígido vazio com a órtese e enchê-lo com líquido, usando a mão } \\
\text { dominante. }\end{array}$ & $\mathbf{x}$ & \\
\hline 4 & $\begin{array}{l}\text { Segurar copo de plástico rígido com líquido com a órtese e misturar açúcar segurando uma } \\
\text { colher com a mão dominante. }\end{array}$ & $\mathbf{x}$ & \\
\hline 5 & $\begin{array}{l}\text { Segurar recipiente com tampa de rosca (creme dental) com a órtese, retirar a tampa com a } \\
\text { mão dominante e colocar creme dental na escova. }\end{array}$ & & $\mathbf{x}$ \\
\hline
\end{tabular}

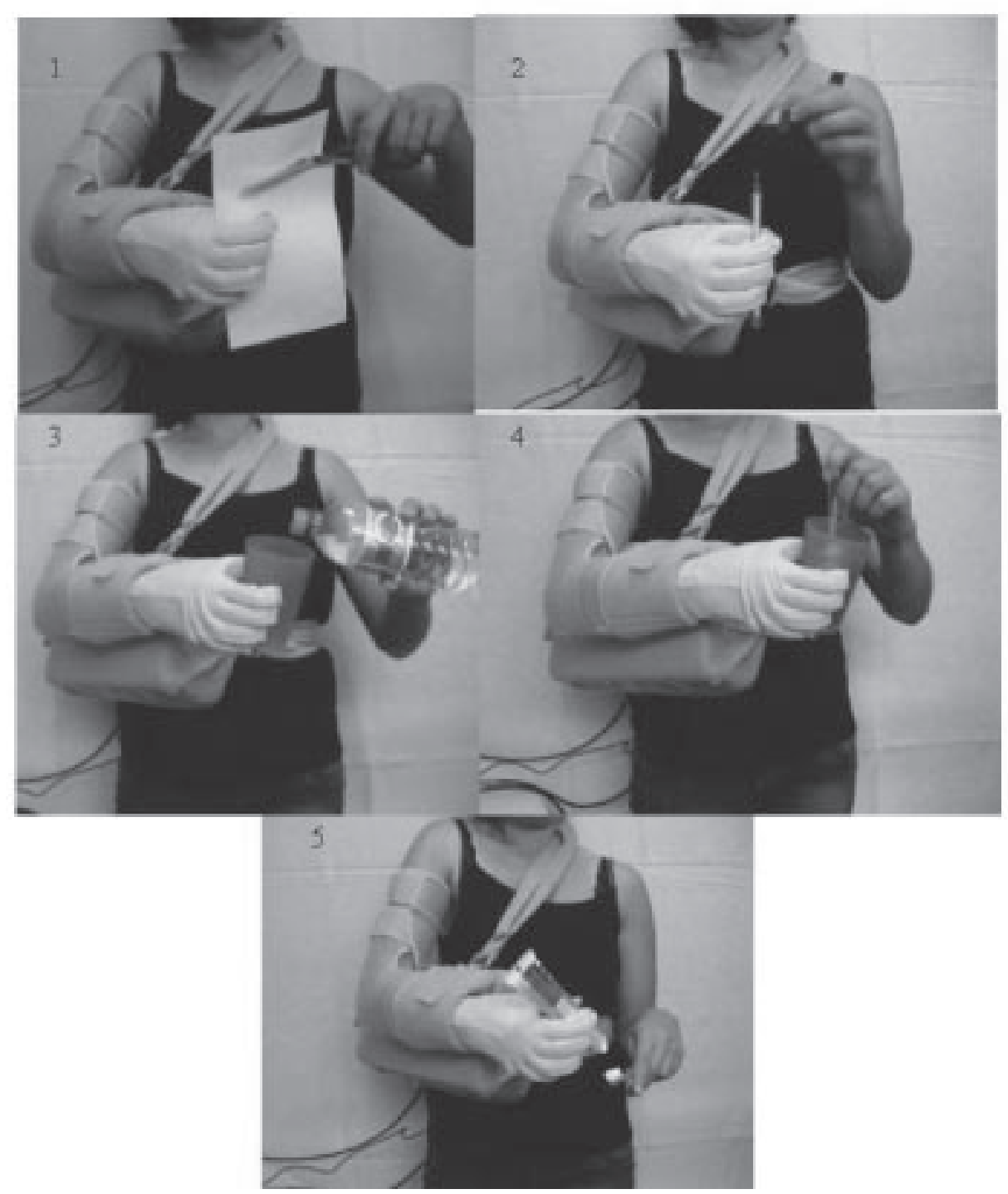

FIGURA 3. Realizando as tarefas do Teste de Desempenho em Atividades Bimanuais 
MENESES, K. V. P. de et al. Aplicação da luva funcional. Rev. Ter. Ocup. Univ. São Paulo, v. 20, n. 2, p. 92-100, maio/ago. 2009.

O terceiro instrumento testado foi o "Questionário de Satisfação", cujo objetivo foi verificar o grau de conforto, funcionalidade e de satisfação da paciente com o uso da Luva Funcional. Em relação aos itens relativos ao conforto, todos os itens obtiveram resposta positiva (SIM), exceto o primeiro item que diz respeito à facilidade na colocação da luva. Em relação aos itens relativos à funcionalidade e à satisfação, todos os itens obtiveram respostas positivas (SIM), como mostra a Tabela 4.

TABELA 4. Resultado do Questionário de Satisfação

\begin{tabular}{|c|c|c|c|}
\hline & Tarefa & Sim & Não \\
\hline 1 & A colocação da Luva Funcional foi realizada com facilidade & & $\mathbf{X}$ \\
\hline 2 & A retirada da Luva Funcional foi realizada com facilidade & $\mathbf{x}$ & \\
\hline 3 & A Luva Funcional é leve & $\mathbf{x}$ & \\
\hline 4 & O barulho da Luva Funcional não incomodou & $\mathbf{x}$ & \\
\hline 5 & Os eletrodos foram corretamente fixados & $\mathbf{X}$ & \\
\hline 6 & O acionamento da Luva Funcional foi realizado com facilidade & $\mathbf{X}$ & \\
\hline 7 & O controle dos movimentos de abertura e fechamento da mão foi realizado com facilidade & $\mathbf{x}$ & \\
\hline 8 & $\begin{array}{l}\text { A Luva Funcional irá melhorar meu desempenho em atividades que antes eu não conseguia } \\
\text { realizar sem ajuda }\end{array}$ & $\mathbf{x}$ & \\
\hline 9 & Eu gostei da aparência da Luva Funcional & $\mathbf{x}$ & \\
\hline 10 & Estou satisfeito com o uso da Luva Funcional & $\mathbf{X}$ & \\
\hline 11 & Recomendo o uso da Luva Funcional para outros pacientes que apresentam paralisia na mão & $\mathbf{X}$ & \\
\hline 12 & Eu gostaria de ter uma da Luva Funcional & $\mathbf{X}$ & \\
\hline
\end{tabular}

\section{CONCLUSÃO}

A Luva Funcional foi capaz de possibilitar os movimentos de flexão e de extensão dos dedos em um indivíduo com paralisia em mão e punho devido à lesão do plexo braquial. O paciente conseguiu controlar a abertura e fechamento da mão por meio do sinal mioelétrico dos músculos selecionados para sua ativação e conseguiu desempenhar, com o uso da órtese Luva funcional, atividades unimanuais e bimanuais. O resultado do Questionário de Satisfação demonstrou satisfação do paciente com o uso da órtese Luva Funcional.

A Luva Funcional pode ser utilizada por indivíduos que não apresentam movimentação ativa nas articulações do punho e dedos. Associada ao uso de uma tipóia ou outro equipamento para posicionar o membro lesado, pode ser utilizada por pacientes apresentando paralisia em todo o membro superior. Como é feita de tecido, é leve e se adapta facilmente sobre a mão do paciente oferecendo proteção, conforto e boa aparência estética. Ela portátil e não interfere na deambulação do paciente. Apresenta facilidade de acionamento e controle.

Estudos futuros com amostras maiores e em pacientes apresentando paralisias na mão devida outras patologias são necessários para verificar a contribuição da Luva Funcional na melhoria da função manual.

Reconhecimento: Esse projeto foi financiado com recursos do Conselho Nacional de Desenvolvimento Científico e Tecnológico- CNPq e da Fundação de Amparo à Pesquisa de Minas Gerais - FAPEMIG. 
MENESES, K. V. P. de et al. Aplicação da luva funcional. Rev. Ter. Ocup. Univ. São Paulo, v. 20, n. 2, p. 92-100, maio/ago. 2009.

MENESES, K. V. P. de; ROCHA, D. N.; CORRÊA, M. F. S.; PINOTTI, M. Application of functional glove in an individual with paralysis of the hand and wrist: a pilot study. Rev. Ter. Ocup. Univ. São Paulo, v. 20, n. 2, p. 92-100, maio./ago. 2009.

\begin{abstract}
One of the interventions used by occupational therapist in the rehabilitation program of individuals with physical dysfunction is orthosis fabrication and prescription. However, hand functional orthoses has proven itself one of the great challenges of the rehabilitation process due to the complexity of the human hand. The motivation for this work was based on the results of in vitro tests performed with the prototype of the Functional Glove, which showed great potential in enabling the extension and flexion of the fingers in patients with hand paralysis. The objective of this study was to investigate the use of Functional Glove in an individual who did not present active movements in hand and wrist. We conducted a pilot study, applying the following instruments: Test for Performing Unimanuals Activities, Test for Performing Bimanuals Activities and a Satisfaction Questionnaire. The patient was able to control the opening and closing the hand through the myoelectric signal of the muscles selected for their activation and managed to play with the orthose Functional Glove, one-hand and bimanual activities. The result of the satisfaction questionnaire showed patient satisfaction with the orthose Functional Glove.
\end{abstract}

KE YWORDS: Self-help devices. Brachial plexus neuropathies/rehabilitation. Technology/ methods. Occupational therapy.

\title{
REFERÊNCIAS
}

ALON, G.; MCBRIDE, K. Person with C5 or C6 tetraplegia achieve seleted functional gains using a neuroprosthesis. Arch. Phys. Med. Rehabil., v. 84, n.1, p.119-124, 2003.

BENJUYA, N.; KENNEY, S. B. Myoelectric hand orthosis. $J$. Prosth. Orthot.. v. 2, n.2, p.149-154, 1990.

HARVEY, L. Principles of conservative management for a Nonorthotic tenodesis grip in tetraplegics. J. Hand Ther, v. 9, n. 3, p. 238-242, 1996.

KILGORE, K. L., et al. Synthesis of hand grasp using functional neuromuscular stimulation. Trans. Biomed. Eng., v.36, n. 7, p.761-770, 1989.

KOHLMEYER, K. M.; WEBER, C. G., YARKONY, G. M. A new orthosis for central cord syndrome and brachial plexus injuries. Arch. Phys. Med. Rehabil, v. 71, p. 1006-1009, 1990.

MENESES, K. V. P. Desenvolvimento de um protótipo de órtese funcional para mão. 2005. Dissertação (Mestrado em Engenharia Mecânica) - Escola de Engenharia, Universidade Federal de Minas Gerais, Belo Horizonte, 2005.

MENESES, K.V. P., et al. Testes preliminaries de um protótipo de órtese funcional para mão. Rev. Ter. Ocup. Univ. São Paulo, v.19, n. 3, p.193-201, 2008.

MENESES, K. V. P.; ROCHA, D. N.; REZENDE, H. M.; CORREA, M. F. S.; SCHULTZ, O. F. L.; BARROSO, P. N.; PINOTTI, M. Development of a functional hand orthosis. In: INTERNATIONAL CONGRESS OF MECHANICAL ENGINEERING, 18. Nov. 6-11, 2005, Ouro Preto, MG.

PERRY, J. et al. Orthoses in patients with braquial plexus injuries. Arch. Phys. Med. Rehabil., v. 55, Mar. 1974.

PETROFF, N. et al. Fuzzy-control of a hand orthosis for restoring tip pinch, lateral pinch, and cylindrical prehensions to patients with elbow flexion intact. IEEE Transact. Neural Systems Rehabil. Eng., v.9, n. 2, p.225-231, 2001.

PINTO, S. A. P. Projeto, implementação e avaliação de uma órtese funcional robotizada de mão. 1999. Dissertação (Mestrado) Departamento de Ciência da Computação UFMG, 1999.

PROCHAZKA, A. et al. The bionic glove: an electrical stimulator garment that provides controlled grasp and hand opening in quadriplegia. Arch. Phys. Med. Rehabil.. v. 78, p.608-614, 1997.

ROCHA, D. N. Desenvolvimento do sistema de controle de uma órtese de mão. 2007. Dissertação (Mestrado em Engenharia 
MENESES, K. V. P. de et al. Aplicação da luva funcional. Rev. Ter. Ocup. Univ. São Paulo, v. 20, n. 2, p. 92-100, maio/ago. 2009.

Mecânica) - Escola de Engenharia, Universidade Federal de Minas Gerais, Belo Horizonte, 2007.

ROCHA, D. N., MENESES, K. V. P.; AFONSO, A. A.; CORRÊA, M. F. S.; BATISTA, C. E. P.; PINOTTI, M. Controle de Força de Preensão para uma Órtese de mão por meio da Análise da Corrente Elétrica do Atuador. Rev. Matéria, v. 12, n. 1, p. 173-178, 2007. Disponível em: <http://www.materia.coppe.ufrj.br/sarra/artigos/ artigo10865>.

ROMILLY, D.P., et al. A functional task: Analysis and motion simulation for the development of a powered upper-limb orthosis. IEEE Trans. Rehabil. Eng., v. 2, n. 3, p. 119-129, sep. 1994.

SLACK, M.; BERBRAYER, D. A myoelectrically controlled wrist-hand orthosis for brachial plexus injury: a case study. $J$. Prosth. Orthotics, v.4, n. 3, p.171-174, 1992.
TROMBLY, C. A, LIDEN, C. A. Orthoses: kinds and purposes. In: TROMBLY, C. A. Occupational therapy for physical dysfunction. 4. ed. Baltimore: Willians \& Willians, 1995.

ROMBLY, C. A.; RADOMSKI, M. V. Occupational therapy for physical dysfunction. 5. ed. Baltimore: Williams \& Wilkins, 2005.

WIERZBICKA, M. M., WIEGNER, A. W. Orthosis for improvement of arm function in $\mathrm{C} 5 / \mathrm{C} 6$ tetraplegia. J. Prosth. Orthotics, v.8, n.3, p. 86-92, 1996.

WUOLLE, K. S. et al. Developmet of a quantitative hand grasp and release test for participants with tetraplegia using a hand neuroposthesis. J. Hand Surg., v.19A, p.209-218, 1994. 